\title{
Conformal symmetries of spherical spacetimes
}

\author{
S. Moopanar • S. D. Maharaj
}

Received: date / Accepted: date

\begin{abstract}
We investigate the conformal geometry of spherically symmetric spacetimes in general without specifying the form of the matter distribution. The general conformal Killing symmetry is obtained subject to a number of integrability conditions. Previous results relating to static spacetimes are shown to be a special case of our solution. The general inheriting conformal symmetry vector, which maps fluid flow lines conformally onto fluid flow lines, is generated and the integrability conditions are shown to be satisfied. We show that there exists a hypersurface orthogonal conformal Killing vector in an exact solution of Einstein's equations for a relativistic fluid which is expanding, accelerating and shearing.
\end{abstract}

Keywords Conformal symmetries · Spherical symmetry · Einstein's equations

\section{Introduction}

Conformal symmetries are studied in general relativity because they preserve the structure of the null cone. Together with conventional isometries, which have been more widely studied, they help to provide a deeper insight into the spacetime geometry. They assist in the generation of exact solutions, sometimes new solutions, of the Einstein field equations which may be utilised to model relativistic, astrophysical and cosmological phenomena. Stephani et al. 1] have emphasized the role of symmetries in classifying and categorising exact solutions. Symmetries are used as one of the principal classification schemes in their catalogue of known solutions. However there are few conformal symmetries known even in spacetimes of high symmetry. Clearly there is a need to study conformal symmetries in a general relativistic setting.

In recent treatments conformal symmetries have been studied by Keane and Barrett [2] in Robertson-Walker spacetimes, Tupper et al. 3 ] in null spacetimes and Keane and Tupper 4 in $p p$-wave spacetimes. We have chosen to investigate the existence of conformal symmetries in inhomogeneous spherically symmetric spacetimes because of

S. Moopanar · S. D. Maharaj

Astrophysics and Cosmology Research Unit, School of Mathematical Sciences, Private Bag X54001, University of KwaZulu-Natal, Durban 4000, South Africa

E-mail: maharaj@ukzn.ac.za 
their physical significance. Our intention is to generate the general conformal Killing vector without making any additional assumptions.

In Sect.2 the line element and the conformal Killing vector equations are presented; the conformal equations are solved in general and the solution can be compactly written subject to integrability conditions. The results of Maharaj et al. [5] are regained for the special case of static spherically symmetric spacetimes in Sect. 3. We find the general inheriting conformal symmetry vector, which maps fluid lines conformally, by fully integrating the integrability conditions in Sect. 4. We demonstrate, in Sect. 5 , that an exact solution of the Einstein field equations for an expanding, shearing and accelerating relativistic fluid admits a hypersurface orthogonal conformal Killing vector.

\section{Conformal equations}

The line element for the general spherically symmetric spacetimes can be written as

$$
d s^{2}=-e^{2 \nu(t, r)} d t^{2}+e^{2 \lambda(t, r)} d r^{2}+Y^{2}(t, r)\left(d \theta^{2}+\sin ^{2} \theta d \phi^{2}\right)
$$

where the functions $\nu, \lambda$ and $Y$ represent the gravitational potentials. In the representation (1) we have used comoving coordinates $\left(x^{a}\right)=(t, r, \theta, \phi)$ relative to the fluid $4-$ velocity $u^{a}=e^{-\nu} \delta_{0}^{a}$. By spherical symmetry the vorticity has to vanish in comoving coordinates. However the acceleration, expansion and the shear are the nonvanishing kinematical quantities in general. In the special case of spherically symmetric spacetimes which are static we can adapt the comoving coordinates to write the line element in the Schwarzschild form

$$
d s^{2}=-e^{2 \nu(r)} d t^{2}+e^{2 \lambda(r)} d r^{2}+r^{2}\left(d \theta^{2}+\sin ^{2} \theta d \phi^{2}\right)
$$

The metric (1) is utilised in constructing inhomogeneous cosmological models (see Stephani et al. 1]) and the metric (2) is used in describing dense stellar astrophysical models (see Delgaty and Lake [6]).

A conformal Killing vector $\mathbf{X}$ is defined by the action of the Lie infinitesimal operator $\mathcal{L}_{\mathbf{X}}$ on the metric tensor field $\mathbf{g}$ so that

$$
\mathcal{L}_{\mathbf{X}} g_{a b}=2 \psi g_{a b}
$$

where $\psi=\psi\left(x^{a}\right)$ is the conformal factor. In this section we analyse the full conformal symmetry for spherically symmetric manifolds without making any assumptions about the conformal vector $\mathbf{X}$ or the conformal factor $\psi$. 
The conformal equation (3) for the metric (1) reduces to the following set of equations

$$
\begin{aligned}
\nu_{t} X^{0}+\nu_{r} X^{1}+X_{t}^{0} & =\psi \\
e^{2 \lambda} X_{t}^{1}-e^{2 \nu} X_{r}^{0} & =0 \\
Y^{2} X_{t}^{2}-e^{2 \nu} X_{\theta}^{0} & =0 \\
Y^{2} \sin ^{2} \theta X_{t}^{3}-e^{2 \nu} X_{\phi}^{0} & =0 \\
\lambda_{t} X^{0}+\lambda_{r} X^{1}+X_{r}^{1} & =\psi \\
Y^{2} X_{r}^{2}+e^{2 \lambda} X_{\theta}^{1} & =0 \\
Y^{2} \sin ^{2} \theta X_{r}^{3}+e^{2 \lambda} X_{\phi}^{1} & =0 \\
\frac{Y_{t}}{Y} X^{0}+\frac{Y_{r}}{Y} X^{1}+X_{\theta}^{2} & =\psi \\
\sin ^{2} \theta X_{\theta}^{3}+X_{\phi}^{2} & =0 \\
\frac{Y_{t}}{Y} X^{0}+\frac{Y_{r}}{Y} X^{1}+\cot \theta X^{2}+X_{\phi}^{3} & =\psi
\end{aligned}
$$

which have been verified with the help of Mathematica. In the above we have used the notation where subscripts denote partial differentiation. The equations (4) comprise a coupled system of partial differential equations which are first order and linear in $\mathbf{X}=\left(X^{0}, X^{1}, X^{2}, X^{3}\right)$ and $\psi$.

The system (4) may be integrated in general to find the components $X^{0}, X^{1}, X^{2}$, $X^{3}$ and $\psi$; this is possible because we can manipulate the system to obtain particular equations involving only the individual components of the vector and the conformal factor. In this process the equations decouple, and a number of integrability conditions (on the metric functions $\nu, \lambda$ and $Y$ ) are generated. It is a simple matter to check that the given solution is valid by direct substitution in (4). The components of the conformal Killing vector $\mathbf{X}$ and the conformal factor $\psi$ are

$$
\begin{aligned}
X^{0}= & Y^{2} e^{-2 \nu} \sin \theta\left(\mathcal{C}_{t} \sin \phi-\mathcal{D}_{t} \cos \phi\right)-Y^{2} e^{-2 \nu} \mathcal{I}_{t} \cos \theta+\mathcal{J} \\
X^{1}= & -Y^{2} e^{-2 \lambda} \sin \theta\left(\mathcal{C}_{r} \sin \phi-\mathcal{D}_{r} \cos \phi\right)+Y^{2} e^{-2 \lambda} \mathcal{I}_{r} \cos \theta+\mathcal{K} \\
X^{2}= & \cos \theta[\mathcal{C} \sin \phi-\mathcal{D} \cos \phi]+\cos \theta\left(a_{1} \sin \phi-a_{2} \cos \phi\right)-a_{3} \sin \phi \\
& +a_{4} \cos \phi+\mathcal{I} \sin \theta \\
X^{3}= & \csc \theta[\mathcal{C} \cos \phi+\mathcal{D} \sin \phi]+\csc \theta\left(a_{1} \cos \phi+a_{2} \sin \phi\right) \\
& -\cot \theta\left(a_{3} \cos \phi+a_{4} \sin \phi\right)+a_{5} \\
\psi= & Y \sin \theta \sin \phi\left[Y e^{-2 \nu} \mathcal{C}_{t t}+\left(2 Y_{t}-Y \nu_{t}\right) e^{-2 \nu} \mathcal{C}_{t}-Y e^{-2 \lambda} \nu_{r} \mathcal{C}_{r}\right] \\
& -Y \sin \theta \cos \phi\left[Y e^{-2 \nu} \mathcal{D}_{t t}+\left(2 Y_{t}-Y \nu_{t}\right) e^{-2 \nu} \mathcal{D}_{t}-Y e^{-2 \lambda} \nu_{r} \mathcal{D}_{r}\right] \\
& -Y \cos \theta\left[Y e^{-2 \nu} \mathcal{I}_{t t}+\left(2 Y_{t}-Y \nu_{t}\right) e^{-2 \nu} \mathcal{I}_{t}-Y e^{-2 \lambda} \nu_{r} \mathcal{I}_{r}\right] \\
& +\mathcal{J}_{t}+\nu_{t} \mathcal{J}+\nu_{r} \mathcal{K}
\end{aligned}
$$

where $\mathcal{A}, \mathcal{C}, \mathcal{D}, \mathcal{I}, \mathcal{J}, \mathcal{K}$ are functions of $t$ and $r$ and $a_{1}-a_{5}$ are constants. This general solution is subject to the following twelve consistency conditions which place 
restrictions on the metric functions:

$$
\begin{aligned}
& Y \mathcal{C}_{t r}+\left(Y_{r}-Y \nu_{r}\right) \mathcal{C}_{t}+\left(Y_{t}-Y \lambda_{t}\right) \mathcal{C}_{r}=0 \\
& Y \mathcal{D}_{t r}+\left(Y_{r}-Y \nu_{r}\right) \mathcal{D}_{t}+\left(Y_{t}-Y \lambda_{t}\right) \mathcal{D}_{r}=0 \\
& Y \mathcal{I}_{t r}+\left(Y_{r}-Y \nu_{r}\right) \mathcal{I}_{t}+\left(Y_{t}-Y \lambda_{t}\right) \mathcal{I}_{r}=0 \\
& Y e^{-2 \nu} \mathcal{C}_{t t}+Y e^{-2 \lambda} \mathcal{C}_{r r}+\left(2 Y_{t}-Y \lambda_{t}-Y \nu_{t}\right) e^{-2 \nu} \mathcal{C}_{t} \\
& +\left(2 Y_{r}-Y \lambda_{r}-Y \nu_{r}\right) e^{-2 \lambda} \mathcal{C}_{r}=0 \\
& Y e^{-2 \nu} \mathcal{D}_{t t}+Y e^{-2 \lambda} \mathcal{D}_{r r}+\left(2 Y_{t}-Y \lambda_{t}-Y \nu_{t}\right) e^{-2 \nu} \mathcal{D}_{t} \\
& +\left(2 Y_{r}-Y \lambda_{r}-Y \nu_{r}\right) e^{-2 \lambda} \mathcal{D}_{r}=0 \\
& Y e^{-2 \nu} \mathcal{I}_{t t}+Y e^{-2 \lambda} \mathcal{I}_{r r}+\left(2 Y_{t}-Y \lambda_{t}-Y \nu_{t}\right) e^{-2 \nu} \mathcal{I}_{t} \\
& +\left(2 Y_{r}-Y \lambda_{r}-Y \nu_{r}\right) e^{-2 \lambda} \mathcal{I}_{r}=0 \\
& Y^{2} e^{-2 \nu} \mathcal{C}_{t t}+Y\left(Y_{t}-Y \nu_{t}\right) e^{-2 \nu} \mathcal{C}_{t}+Y\left(Y_{r}-Y \nu_{r}\right) e^{-2 \lambda} \mathcal{C}_{r}+\mathcal{C}+a_{1}=0 \\
& Y^{2} e^{-2 \nu} \mathcal{D}_{t t}+Y\left(Y_{t}-Y \nu_{t}\right) e^{-2 \nu} \mathcal{D}_{t}+Y\left(Y_{r}-Y \nu_{r}\right) e^{-2 \lambda} \mathcal{D}_{r}+\mathcal{D}+a_{2}=0 \\
& Y^{2} e^{-2 \nu} \mathcal{I}_{t t}+Y\left(Y_{t}-Y \nu_{t}\right) e^{-2 \nu} \mathcal{I}_{t}+Y\left(Y_{r}-Y \nu_{r}\right) e^{-2 \lambda} \mathcal{I}_{r}+\mathcal{I}=0 \\
& e^{2 \lambda} \mathcal{K}_{t}-e^{2 \nu} \mathcal{J}_{r}=0 \\
& -\mathcal{J}_{t}+\left(\frac{Y_{t}}{Y}-\nu_{t}\right) \mathcal{J}+\left(\frac{Y_{r}}{Y}-\nu_{r}\right) \mathcal{K}=0 \\
& -\mathcal{J}_{t}+\mathcal{K}_{r}+\left(\lambda_{t}-\nu_{t}\right) \mathcal{J}+\left(\lambda_{r}-\nu_{r}\right) \mathcal{K}=0
\end{aligned}
$$

Note that in our solution the angular dependence in $\theta$ and $\phi$ is known explicitly. This feature of the solution is not surprising as the spacetime is spherically symmetric. There is freedom only in the $t$ and $r$ coordinates.

The above solution may be expressed more compactly if we utilise the notation adopted by Maartens et al. 7 in their categorisation of static spacetimes. We first let

$$
\mathcal{C}+a_{1}=\tilde{\mathcal{C}} \quad \text { and } \quad \mathcal{D}+a_{2}=\tilde{\mathcal{D}}
$$

and introduce the new variables

$$
\begin{aligned}
A^{i} & =\left(A^{1}, A^{2}, A^{3}\right)=(\tilde{\mathcal{C}}(t, r),-\tilde{\mathcal{D}}(t, r),-\mathcal{I}(t, r)) \\
\eta_{i} & =\left(\eta_{1}, \eta_{2}, \eta_{3}\right)=(\sin \theta \sin \phi, \sin \theta \cos \phi, \cos \theta) \\
A^{0} & =\mathcal{J}(t, r) \\
A^{4} & =\mathcal{K}(t, r)
\end{aligned}
$$

The components of the conformal Killing vector and the conformal factor then become

$$
\begin{aligned}
X^{0}= & Y^{2} e^{-2 \nu} A_{t}^{i} \eta_{i}+A^{0} \\
X^{1}= & -Y^{2} e^{-2 \lambda} A_{r}^{i} \eta_{i}+A^{4} \\
X^{2}= & A^{i}\left(\eta_{i}\right)_{\theta}-a_{3} \sin \phi+a_{4} \cos \phi \\
X^{3}= & \csc ^{2} \theta A^{i}\left(\eta_{i}\right)_{\phi}-\cot \theta\left(a_{3} \cos \phi+a_{4} \sin \phi\right)+a_{6} \\
\psi= & Y \eta_{i}\left[Y e^{-2 \nu} A_{t t}^{i}+\left(2 Y_{t}-Y \nu_{t}\right) e^{-2 \nu} A_{t}^{i}-Y e^{-2 \lambda} \nu_{r} A_{r}^{i}\right]+A_{t}^{0}+\nu_{t} A^{0} \\
& +\nu_{r} A^{4}
\end{aligned}
$$


The consistency conditions simplify to

$$
\begin{aligned}
Y A_{t r}^{i}+\left(Y_{r}-Y \nu_{r}\right) A_{t}^{i}+\left(Y_{t}-Y \lambda_{t}\right) A_{r}^{i} & =0 \\
Y e^{-2 \nu} A_{t t}^{i}+Y e^{-2 \lambda} A_{r r}^{i}+\left(2 Y_{t}-Y \lambda_{t}-Y \nu_{t}\right) e^{-2 \nu} A_{t}^{i} & \\
+\left(2 Y_{r}-Y \lambda_{r}-Y \nu_{r}\right) e^{-2 \lambda} A_{r}^{i} & =0 \\
Y^{2} e^{-2 \nu} A_{t t}^{i}+Y\left(Y_{t}-Y \nu_{t}\right) e^{-2 \nu} A_{t}^{i}+Y\left(Y_{r}-Y \nu_{r}\right) e^{-2 \lambda} A_{r}^{i}+A^{i} & =0 \\
e^{2 \lambda} A_{t}^{4}-e^{2 \nu} A_{r}^{0} & =0 \\
-A_{t}^{0}+\left(\frac{Y_{t}}{Y}-\nu_{t}\right) A^{0}+\left(\frac{Y_{r}}{Y}-\nu_{r}\right) A^{4} & =0 \\
-A_{t}^{0}+\left(\lambda_{t}-\nu_{t}\right) A^{0}+\left(\lambda_{r}-\nu_{r}\right) A^{4}+A_{r}^{4} & =0
\end{aligned}
$$

It is remarkable that the general conformal symmetry (5) can be generated explicitly since the line element (1) represents the most general spherically symmetric spacetime. The spacetime may be nonstatic, accelerating, expanding and shearing. The integrability conditions (6) simplify or can be completely solved depending on the forms of the potentials $\nu, \lambda$ and $Y$. We emphasise that this is the most general solution to the conformal Killing vector equation (3) for the spherically symmetric spacetime (1). In the following sections we consider some particular cases of this solution and their relationship to exact solutions of the Einstein field equations.

\section{Static spacetimes}

To regain the static spherically symmetric spacetimes (2) we set

$$
\nu=\nu(r), \quad \lambda=\lambda(r), \quad Y=r
$$

Then the components of the conformal Killing vector and the conformal factor (5) become

$$
\begin{aligned}
X^{0} & =r^{2} e^{-2 \nu} A_{t}^{i} \eta_{i}+A^{0} \\
X^{1} & =-r^{2} e^{-2 \lambda} A_{r}^{i} \eta_{i}+A^{4} \\
X^{2} & =A^{i}\left(\eta_{i}\right)_{\theta}-a_{3} \sin \phi+a_{4} \cos \phi \\
X^{3} & =\csc ^{2} \theta A^{i}\left(\eta_{i}\right)_{\phi}-\cot \theta\left(a_{3} \cos \phi+a_{4} \sin \phi\right)+a_{6} \\
\psi & =r^{2} \eta_{i}\left(e^{-2 \nu} A_{t t}^{i}-e^{-2 \lambda} \nu^{\prime} A_{r}^{i}\right)+A_{t}^{0}+\nu^{\prime} A^{4}
\end{aligned}
$$

The integrability conditions (6) simplify to

$$
\begin{aligned}
r A_{t r}^{i}+\left(1-r \nu^{\prime}\right) A_{t}^{i} & =0 \\
r e^{-2 \nu} A_{t t}^{i}+r e^{-2 \lambda} A_{r r}^{i}+\left(2-r \lambda^{\prime}-r \nu^{\prime}\right) e^{-2 \lambda} A_{r}^{i} & =0 \\
r^{2} e^{-2 \nu} A_{t t}^{i}+r\left(1-r \nu^{\prime}\right) e^{-2 \lambda} A_{r}^{i}+A^{i} & =0 \\
e^{2 \lambda} A_{t}^{4}-e^{2 \nu} A_{r}^{0} & =0 \\
-A_{t}^{0}+\left(\frac{1}{r}-\nu^{\prime}\right) A^{4} & =0 \\
-A_{t}^{0}+\left(\lambda^{\prime}-\nu^{\prime}\right) A^{4}+A_{r}^{4} & =0
\end{aligned}
$$

where primes denote differentiation with respect to $r$. 
We observe that the equations (7)-(8) are equivalent to the corresponding system obtained by Maharaj et al. [5]. Maartens et al. [7,8] demonstrated that the conformal solution (7)-(8) can be used for categorisation and classification of static spherically symmetric spacetimes in terms of the Weyl tensor. A number of exact solutions to the Einstein field equations with a conformal symmetry, corresponding to special cases of (17) -(8), have been found. These include the solutions of Coley and Tupper [9,10, 11, Herrera et al. 12, Herrera and Ponce de Leon 13, Maartens and Maharaj 14, Mak and Harko [15] and Tello-Llanos [16].

\section{Inheriting vectors}

Herrera et al. 12 and Maartens et al. 17] introduced the condition

$$
\mathcal{L}_{\mathbf{X}} u_{a}=\psi u_{a}
$$

where $\mathbf{u}$ is the fluid four-velocity. Coley and Tupper 9 called vectors satisfying (9) inheriting conformal Killing vectors as fluid flow lines are mapped conformally. The existence of inheriting vectors in spherically symmetric spacetimes has been comprehensively studied by Coley and Tupper 10,11 for particular forms of the energy momentum tensor and gravitational potentials. Here we show that it is possible to generate the general inheriting conformal Killing vector for the spacetime (1) from our conformal solution (5)-(6). This solution will be applicable to any matter distribution.

In a comoving frame of reference the fluid 4-velocity $\mathbf{u}$ is given by $u^{a}=e^{-\nu} \delta_{0}^{a}$. On substitution in the inheriting condition (9) we obtain the following system:

$$
\begin{aligned}
\nu_{t} X^{0}+\nu_{r} X^{1}+X_{t}^{0} & =\psi \\
X_{r}^{0} & =0 \\
X_{\theta}^{0} & =0 \\
X_{\phi}^{0} & =0
\end{aligned}
$$

It is relatively straight-forward to show that the integrability conditions (6) and the inheriting conditions (10) may be solved in general. This essentially follows because (10) implies that $X^{0}=X^{0}(t)$ and the integration is simplified. The components of the inheriting conformal vector and the conformal factor are

$$
\begin{aligned}
X^{0} & =A^{0}(t) \\
X^{1} & =A^{4}(r) \\
X^{2} & =-a_{3} \sin \phi+a_{4} \cos \phi \\
X^{3} & =-\cot \theta\left(a_{3} \cos \phi+a_{4} \sin \phi\right)+a_{6} \\
\psi & =\dot{A}^{0}+\nu_{t} A^{0}(t)+\nu_{r} A^{4}(r)
\end{aligned}
$$

and the integrability conditions ([6) reduce to

$$
\begin{aligned}
-\dot{A}^{0}+\left(\frac{Y_{t}}{Y}-\nu_{t}\right) A^{0}+\left(\frac{Y_{r}}{Y}-\nu_{r}\right) A^{4} & =0 \\
-\dot{A}^{0}+\left(A^{4}\right)^{\prime}+\left(\lambda_{t}-\nu_{t}\right) A^{0}+\left(\lambda_{r}-\nu_{r}\right) A^{4} & =0
\end{aligned}
$$

Note that the inheriting condition (10a) is identically satisfied with the $X^{0}, X^{1}$ and $\psi$ given in (11). 
The integrability equations (12) comprise a system of first order partial differential equations which may be solved using the method of characteristics. The general solution is given by

$$
\begin{aligned}
\ln Y-\nu & =F(u)+\ln A^{0} \\
\lambda-\nu & =F(u)-G(u)+\ln A^{0}-\ln A^{4}
\end{aligned}
$$

where

$$
u=\int \frac{d t}{A^{0}}-\int \frac{d r}{A^{4}}
$$

and $F, G$ are arbitrary functions. Thus we have generated the general inheriting conformal symmetry (11) for the spherically symmetric line element (11). The metric functions $\nu, \lambda$ and $Y$ are restricted by (13) for the existence of such a symmetry. Of course the form of this inheriting vector will be restricted further on application of the field equations.

It is important to note that spacetimes admitting inheriting Killing vectors are very rare. This follows from the detailed analyses of Coley and Tupper 9, 10. They demonstrated that orthogonal synchronous, perfect fluid spacetimes do not admit any proper inheriting Killing vectors; the Robertson-Walker spacetimes are the exceptional case that admit inheriting vectors. The spherically symmetric spacetimes with known examples of inheriting vectors are the special cases of conformal Robertson-Walker, static Schwarzschild interior spacetimes and the generalised Gutman-Bespalko-Wesson spacetimes. In this section we have found the general form of the inheriting conformal symmetry for the shearing spherically symmetric spacetime (1). This generalises the results of Coley and Tupper. The generation of the inheriting symmetry (11) is made possible by the general conformal symmetry (5)-(6).

\section{Exact solutions with conformal symmetry}

It is important to characterise exact solutions of the Einstein field equations with a symmetry vector so that the geometrical features can be studied. For the general spherically symmetric metric (1), the consistency conditions (6) have to be solved in conjunction with the nonlinear field equations to generate the conformal vector $\mathbf{X}$. The nonlinearity of the Einstein equations makes this a formidable task to achieve in general. If we set $\nu=\nu(r), \lambda=\lambda(r)$ and take $Y(t, r)$ to be a separable function then the Einstein field equations can be solved to admit the line element

$$
d s^{2}=-\frac{r^{2}}{4} d t^{2}+\frac{1}{\varepsilon+c r^{2}} d r^{2}+r^{2}\left(\frac{\varepsilon}{2}+h(t)\right)\left(d \theta^{2}+\sin ^{2} \theta d \phi^{2}\right)
$$

where

$$
h(t)= \begin{cases}A \sin t+B \cos t & \varepsilon=-1 \\ -\frac{1}{4} t^{2}+A t+B & \varepsilon=0 \\ A e^{t}+B e^{-t} & \varepsilon=1\end{cases}
$$

with $A, B, \varepsilon$ and $c$ being constants. A number of authors (see Stephani et al. 1]) have shown that the expanding, accelerating and shearing spacetime (14) admits the barotropic equation of state $p=\mu+6 c$. Maharaj and Maharaj 18] have shown that 
the class of spherically symmetric solutions (14) admits a hypersurface orthogonal conformal Killing vector $\mathbf{X}=\left(0, X^{1}, 0,0\right)$ where

$$
X^{1}= \begin{cases}D_{-} r\left(-1+c r^{2}\right)^{1 / 2} & \varepsilon=-1 \\ D_{0} r^{2} & \varepsilon=0 \\ D_{+} r\left(1+c r^{2}\right)^{1 / 2} & \varepsilon=1\end{cases}
$$

and conformal factor

$$
\psi= \begin{cases}D_{-}\left(-1+c r^{2}\right)^{1 / 2} & \varepsilon=-1 \\ D_{0} r & \varepsilon=0 \\ D_{+}\left(1+c r^{2}\right)^{1 / 2} & \varepsilon=1\end{cases}
$$

where $D_{-}, D_{0}$ and $D_{+}$are constants. (Note that the final forms of $\mathbf{X}$ and $\psi$ in the Maharaj and Maharaj [18] paper contain errors which we have corrected in this solution). It is easy to verify that the consistency conditions (6) are identically satisfied with this form of $\mathbf{X}$. The special conformal symmetry $\mathbf{X}=\left(0, X^{1}, 0,0\right)$ arises because of the simple, separable form of the metric (14). The existence of this radial conformal symmetry suggests that a more detailed study of solutions of Einstein equations, invariant under the action of the conformal vector $\mathbf{X}$, should be pursued. In future work, we intend to investigate the compatibility of the conformal symmetry with homogeneity, and also with the kinematical quantities (shear, expansion, acceleration) following the treatment of Lortan et al. [19.

Acknowledgements SM thanks the National Research Foundation and the University of KwaZulu-Natal for financial support. SDM acknowledges that this work is based upon research supported by the South African Research Chair Initiative of the Department of Science and Technology and the National Research Foundation.

\section{References}

1. Stephani, H., Kramer, D., MacCallum, M., Hoenselaers, C., Herlt, E.: Exact solutions to Einstein's field equations, Cambridge University Press, Cambridge (2003)

2. Keane, A. J., Barrett, R. K.: Class. Quantum Grav. 17, 201 (2000)

3. Tupper, B. O. J., Keane, A. J., Hall, G. S., Coley, A. A., Carot, J.: Class. Quantum Grav. 20, 801 (2003)

4. Keane, A. J., Tupper, B. O. J.: Class. Quantum Grav. 21, 2037 (2004)

5. Maharaj, S. D., Maartens, R., Maharaj, M. S.: Int. J. Theor. Phys. 34, 2285 (1995)

6. Delgaty, M. S. R., Lake, K.: Comput. Phys. Commun. 115, 395 (1998)

7. Maartens, R., Maharaj, S. D., Tupper, B. O. J.: Class. Quantum Grav. 12, 2577 (1995)

8. Maartens, R., Maharaj, S. D., Tupper, B. O. J.: Class. Quantum Grav. 13, 317 (1996)

9. Coley, A. A., Tupper, B. O. J.: Class. Quantum Grav. 7, 1961 (1990)

10. Coley, A. A., Tupper, B. O. J.: Class. Quantum Grav. 7, 2195 (1990)

11. Coley, A. A., Tupper, B. O. J.: Class. Quantum Grav. 11, 2553 (1994)

12. Herrera, L., Jimenez, J., Leal, L., Ponce de Leon, J., Esculpi, M., Galina, V.: J. Math.

Phys. 25, 3274 (1984)

13. Herrera, L., Ponce de Leon, J.: J. Math. Phys. 26, 2302 (1985)

14. Maartens, R., Maharaj, M. S.: J. Math. Phys. 31, 151 (1990)

15. Mak, M. K., Harko, T.: Int. J. Mod. Phys. D 13, 273 (2004)

16. Tello-Llanos, R. A.: Gen. Relativ. Gravit. 20, 765 (1988)

17. Maartens, R., Mason, D. P., Tsamparlis, M.: J. Math. Phys. 27, 2987 (1986)

18. Maharaj, S. D., Maharaj, M. S.: Il Nuovo Cimento B 109, 983 (1994)

19. Lortan, D. B., Maharaj, S. D., Dadhich, N. K.: Pramana - J. Phys. 56, 715 (2001) 